\title{
ZUR INTERAKTIVEN BEHANDLUNG VON DEUTUNGEN IN THERAPIEGESPRÄCHEN
}

\author{
Walther KINDT *
}

This paper deals with a new perspective in semantic research. On the one hand it will be argued that meaning constitution can often be regarded as an interactive negotiating process, on the other hand it will be demonstrated that interpreting activities in therapeutic discourse are regulated by specific procedures. These insights have also important consequences for the analysis of therapeutic communication.

\section{Vorbemerkungen}

Der vorliegende Beitrag beschäftigt sich mit der Frage, wie die Teilnehmer von Therapiegesprächen mit Deutungen umgehen. Daß ein Vertreter der logischen Semantik, der ich mich zurechne, ein derartiges Thema bearbeitet, bedarf der Erläuterung. In anderen Arbeiten habe ich meine Auffassung genauer dargestellt, daß dic Fortentwicklung der linguistischen Teildisziplin Semantik insbesondere durch das bestehende Empiriedefizit stagniert (cf. z.B. Kindt (1984a, 1983, 1984b)). Eine der Möglichkeiten, empirische Information über semantische Prozesse zu gewinnen, wird durch die Konversationsanalyse eröffnet. Untersuchungen im Rahmen der Konversationanalyse bzw. der zugrundeliegenden Interaktionsforschung haben nämlich deutlich gemacht, daß Bedeutungen teilweise über konventionelle Anteile hinaus erst im Vollzug der Kommunikation selbst konstituiert werden. Folglich ergibt sich durch die Analyse der zugehörigen interaktiven Konstitutionsprozesse ein empirischer Zugang zu Bedeutungen. Allerdings werden die Interaktionsverfahren der Bedeutungskonstitution aufgrund der großen Routine der Kommunikationsteilnehmer zumeist in stark abgekürzter Form eingesetzt, so daß sie dem

\footnotetext{
* Author's address: Walther Kindt, Fakultät für Linguistik und Literaturwissenschaft, Universität Bielefeld, Postfach 8640, D-4800 Bielefeld, F.R.G.

Der vorliegende Aufsatz ist die gekürzte und üherarheitete Fassung eines Vortragsmanuskripts anlässlich der 'Balint-Tage', Universität Tübingen, Oktober 1981.
} 
analysierenden Linguisten nicht in der wünschenswerten Explizitheit im Datenmaterial vorliegen. Solange man also noch wenig über diese Verfahren weiß, ist es zweckmäßig, solche Gespräche zu untersuchen, bei denen aufgrund von kontextuellen Sonderbedingungen eine größere Explizitheit der Bedeutungskonstitutionsverfahren notwendig ist. Zu diesem Materialtyp gehört auch die therapeutische Kommunikation, wo - in gewissem Sinne - besondere Interpretationsleistungen der Beteiligten erwartet und auch erbracht werden. Von daher erklärt sich zunächst grundsätzlich mein Interesse an Therapiegesprächen.

Weiterhin ist zu erläutern, inwiefern Deutungen der Forschungsgegenstand einer empirischen Semantik sein können. Dazu muß als erstes der intendierte Deutungsbegriff präzisiert werden. Für den im vorliegenden Beitrag entwickelten Zusammenhang ist es zweckmäßig, der Konzeption des symbolischen Interaktionismus $\mathrm{zu}$ folgen und einen generalisierten Interpretations- und Redeutungshegriff zu verwenden: nicht nur sprachliche Äußerungen sondern jeder Gegenstand kann interpretiert werden und das Resultat von Interpretationen ist jeweils eine Bedeutung. Unter Deutung soll nun die Interpretation eines nichtsprachlichen Gegenstandes verstanden werden, wobei die Mehrdeutigkeit bezüglich Prozeß- und Resultatlesart von 'Deutung' beibehalten werden soll, sofern dies zu keinem Mißverständnis führt. Die Besonderheit der Interpretationsleistungen in Therapiegesprächen bezieht sich primär auf die dort vorgenommenen Deutungen und zwar im wesentlichen auf Deutungen der von Klienten bzw. Patienten dargestellten Sachverhalte. Dann ist allerdings zu fragen, worin das spezifische Interesse des Semantikers an therapeutischer Kommunikation bestehen kann, wenn sein Aufgabengebiet doch in der Untersuchung von Bedeutungen sprachlicher Äußerungen liegt. Meine Antwort auf diese Frage basiert auf der Annahme bzw. der bereits gewonnenen Analyseerfahrung, daß die Interpretation von sprachlichen wie von nichtsprachlichen Gegenständen teilweise durch dieselben Interaktionsverfahren gesteuert werden; dies gilt z.B. für die Begründung und Angleichung von Bedeutungen. Insofern trägt die Untersuchung der interaktiven Behandlung von Deutungen auch zu Erkenntnissen über Bedeutungskonstitutionsverfahren für sprachliche Äußerungen bei, die für den Semantiker von Belang sind. Besondere Interpretationsleistungen sind in Therapiegesprächen sekundär auch für die Interpretation der sprachlichen Äußungen zu erwarten. So muß etwa der Therapeut ggf. größere Anstrengungen als in anderen Kontexten unternehmen, um die Mitteilungsintentionen des Klienten richtig zu verstehen, wenn seine Deutungen nicht auf falschen Informationsvoraussetzungen basieren sollen. Und umgekehrt ist zu erwarten, daß sich der Klient in besonderem Maße darum bemüht, die Äußerungen des Therapeuten zu verstehen, weil er sich Hilfestellungen von ihnen erhofft. In diesem Sinne entsteht also in therapeutischer Kommunikation eine Situation, wo eventuell eine stärkere Explizierung zugrundeliegender Interpretationsverfahren erforderlich wird. 


\section{Deutungen}

Die bisherigen Erläuterungen zum Deutungskonzept reichen nicht für die Skizzierung eines Untersuchungsrahmens aus und sollen daher durch einige zusätzliche Überlegungen ergänzt werden.

Für eine weitere Klärung des Deutungskonzepts ist es zweckmäßig, sich anhand typischer Beispiele die Charakteristika von Deutungen zu vergegenwärtigen. Wenn z.B. von Freud (1961: 295) die in Träumen von Männern vorkommende Krawatte unter bestimmten Bedingungen als Penis gedeutet wird, dann wird einerseits von einer u.a. über Formähnlichkeit definierten Abstraktionsoperation Gebrauch gemacht und andererseits eine auf der zugrundeliegenden psychologischen Theorie basierende Ebenenübertragung (Alltagsgegenstände-Sexualgegenstände) vorgenommen. Oder wenn ein Therapeut aus der Erzählung seines Patienten über ein Jugenderlebnis auf die Art der Beziehung des Patienten zu seiner Mutter zurückschließt, dann kategorisiert er unter Rückgriff auf sein psychologisches Wissen das spezielle Erlebnis als Realisierung einer generelleren Konstellation und leitet hieraus Aussagen über Beziehungseigenschaften ab. Beiden Beispielen liegt dasselbe Verarbeitungsmuster zugrunde: In einem ersten Schritt wird ein Gegenstand unter kontextabhängiger und theoriegeleiteter Abstraktion kategorisiert, im zweiten Schritt werden auf der Basis der Kategorisierung theoretische Folgerungen gezogen. Ich will hier nicht behaupten, daß mit den beiden Verarbeitungsschritten von Abstraktion und Folgerung bereits ein generell gültiges Muster für Deutungen gefunden ist; vielmehr geht es mir einerseits um die Markierung einer Richtung für die Explikation des Deutungskonzepts und andererseits um den Hinweis, daß die beiden genannten Verarbeitungsoperationen offensichtlich eine zentrale Rolle spielen. Ein für die Untersuchung des interaktiven Umgangs mit Deutungen wichtiger Aspekt ist darin zu sehen, daß verschiedene Deutungen desselben Gegenstandes im Hinblick auf ihre 'Stärke' miteinander verglichen werden können. Intuitiv beurteilt wird man zunächst nur dann von einer Deutung sprechen, wenn sie durch nichttriviale Abstraktions- und Folgerungsprozesse hervorgegangen ist; in diesem Sinne würde man es beispielsweise keine Deutung nennen, wenn ein Therapeut die Informationen des Klienten nur wiederholt. Aber auch Folgerungen, die auf einem zwischen Therapeut und Klient gemeinsamen Standardwissen beruhen, begründen keinen Anspruch auf Einstufung als Deutung (wenn ein Klient berichtet: 'Gestern habe ich eine Katze gesehen', dann wird man für die etwaige Reaktion: 'Sie haben ein in Europa vorkommendes Säugetier gesehen' im allgemeinen keinen Deutungsanspruch stellen). An dem letzten Beispiel kann man sich zugleich klar machen, daß zu der Forderung nach Nichttrivialität von Deutungen noch die Forderung nach Relevanz hinzukommt. Beide Beurteilungskriterien sind allerdings auf die Kommunikationsteilnehmer zu beziehen und können dementsprechend zu unterschiedlichen Einschätzun- 
gen führen. So erwartet der Klient von der Therapie Deutungen, die für ihn relevant sind und die er aufgrund seiner Wahrnehmung und seines Wissens nicht ohne weiteres selbst hätte produzieren können; die Einstufung der Relevanz von Deutungen kann demgegenüber beim Therapeuten neben dem Therapieziel z.B. auch durch Forschungsinteressen bestimmt sein und gewisse vom Therapeuten gegebene Deutungen werden für ihn selbst bereits trivial sein, weil er sie ihrem Typ nach bereits sehr häufig benutzt hat. An den Konzepten der Relevanz und Stärke von Deutungen lassen sich noch sehr viele Probleme von Therapiegesprächen festmachen; erwähnen möchte ich hier nur, daß Relevanzgrad und Stärkegrad von Deutungen möglicherweise als zueinander proportional eingeschätzt werden, daß mit Stärke oder Relevanz von Deutungen ggf. der Widerstand des Klienten gegen sie wächst, und daß Änderungen der Relevanzeinschätzung durch den Klienten in der Zeit eine wichtige Rolle spielen.

Für die Untersuchung des interaktiven Umgangs mit Deutungen ist ferner die Frage wichtig, in welcher Form Deutungen in der Kommunikation vorkommen. Einen direkten empirischen Zugang besitzt man zu ihnen nur, wenn sie innerhalb von Äußerungen mehr oder weniger explizit manifestiert werden. Eine solche Manifestation liegt vor, wenn die Deutungen in Form von Behauptungen oder Vermutungen geäußert werden. Weniger explizite Formen bestehen darin, daß Deutungen durch nichtproblematisierte Präsuppositionen eingeführt werden oder daß sie durch bestimmte Fragetechniken provoziert, selber aber nicht ausgesprochen werden. Für die Erkennung und Rekonstruktion aller impliziten Formen von Deutungen bestehen die besonderen Schwierigkeiten darin, daß einerseits aus materialtechnischen Gründen für die Analyse nur Gesprächsausschnitte zur Verfügung stehen und damit die von dem Teilnehmern bereits erreichte Verständigungsbasis eventuell nicht nachvollziehbar ist, daß aber andererseits gegebenenfalls auch kein ausreichendes Wissen über die individuellen Hintergrundstheorien, die zur Deutung herangezogen werden, zur Verfügung steht. Speziell bei der Therapieform der Psychoanalyse hat man damit zu rechnen, daß Deutungen zur Vermeidung von Widerständen weitgehend implizit bleiben oder nur langfristig durchgesetzt werden.

Die im vorigen Abschnitt eingeführte Unterscheidung zwischen Äußerungsinterpretationen und Deutungen (z.B. von mitgeteilten Sachverhalten) ist empirisch nicht ohne weiteres auffindbar und darf natürlich insbesondere nicht so verstanden werden, daß bei Interpretationen von Äußerungen in einem zeitlich ersten Schritt die durch Sprachkonventionen bestimmten Bedeutungsanteile ermittelt und in einem zeitlich zweiten Schritt darauf basierende Deutungen vorgenommen werden. Vielmehr müssen Deutung und sprachliche Interpretation als einander abwechselnde oder parallel erfolgende, sich aber in jedem Fall wechselseitig beeinflussende Komponenten eines Interpretationsprozesses gesehen werden. Im allgemeinen sind auch Kommunikationsteilnehmer selbst 
nicht in der Lage, einzelne Interpretationsanteile entsprechend zu verrechnen. Gleichwohl kann man sich anhand von 'klaren' Fällen verdeutlichen, daß die vorgeschlagene Unterscheidung empirisch notwendig ist und daß Kommunikationsteilnehmer z.B. bei Auftreten von Verständigungsproblemen gegebenenfalls auch eine derartige Unterscheidung machen (cf. Kind und Weingarten (1984)). Der Grund für die Notwendigkeit dieser Unterscheidung ist leicht einzusehen: wenn beispielsweise ein Kommunikationsteilnehmer die Äußerung eines anderen Teilnehmers nicht verstanden hat, dann ist es zur raschen Behebung des Verstehenproblems wichtig, mögliche Ursachen zu benennen; die anzuwendende Lösungsstrategie sieht aber in dem Fall, wo dem Hörer nur ein Wort der Äußerung unbekannt ist, ganz anders aus, als in dem Fall, wo er einen für das Verständnis erforderlichen sachlichen Zusammenhang nicht kennt.

\section{Aushandlung und Angleichung}

In der Interaktion ist es generell für das Erreichen gemeinsam gesetzter Ziele notwendig, daß die Interaktionspartner hinsichtlich bestimmter Dinge wie z.B. Wissensvoraussetzungen, Einstellungen etc. gleiche oder ähnliche Positionen haben. Daher müssen sie, falls sich ihre Positionen noch zu stark voneinander unterscheiden, ggf. versuchen, ihre Positionen einander anzugleichen. Wenn beispielsweise zwei Interaktanden vereinbart haben, daß sie am Abend gemeinsam ausgehen wollen, dann müssen sie sich zusätzlich darüber einigen, ob sie etwa ins Kino, ins Theater oder in ein Lokal gehen wollen, und sie müssen weiterhin festlegen, ob sie den Zielort mit dem Auto, der Straßenbahn oder zu Fuß erreichen wollen etc. Dabei werden sie möglicherweise unterschiedliche Präferenzen haben, und es ist die Frage, ob und wie sie zu einer Einigung kommen können. Wenn eine Einigung nicht möglich ist, dann werden sie ihr ursprüngliches Ziel, miteinander auszugehen, fallen lassen müssen. Im gleichen Sinne kann man im Bereich der therapeutischen Kommunikation Einigungsprozesse studieren, die sich auf Deutungen insbesondere des Therapeuten beziehen; dabei ist der Grund für derartige Einigungsversuche durch das globale Therapieziel institutionell vorgegeben.

Für die Angleichung von Positionen gibt es eigene interaktive Verfahren. Sie beziehen sich einerseits auf die Herstellung einer hinreichenden Übereinstimmung der Positionen selbst und andererseits auf den Umgang mit nicht beseitigbaren Differenzen. Eine bekannte Technik für den letzteren Punkt besteht z.B. in einer geeigneten Relevanzeinstufung: Die Interaktanten erklären die Differenzen für unwichtig und heben demgegenüber die erreichte Übereinstimmung als besonders wertvoll hervor. Eine andere Technik besteht darin, daß die Positionsdifferenzen zwar als sehr hedauerlich und als hinderlich für ein ursprünglich gemeinsames Ziel eingeschätzt werden, daß aber die 
Interaktionspartner ihre Zufriedenheit darüber äußern, die Position ihres Partners kennengelernt und aus dessen Perspektive heraus auch verstanden zu haben. Für die Herstellung gemeinsamer Positionen ist in der Literatur das Verfahren der 'Aushandlung' bekannt und es wird von vielen Autoren als zentral angesehen. Erstaunlicherweise sind aber bisher so gut wie keine Versuche unternommen worden, das Aushandlungskonzept genauer zu explizieren bzw. die für Aushandlungen typischen Verfahrensschritte zu bestimmen. Wenn man sich jedoch mit dieser Aufgabe befaßt, dann wird schnell deutlich, daß die 'Griffigkeit' des Aushandlungskonzepts leicht über die zugrundeliegenden Explikationsschwierigkeiten hinwegtäuscht. Erstens ist es immer ein sehr mühsames Unterfangen, ein generelles Interaktionsmuster empirisch adäquat zu charakterisieren. Zweitens treten bestimmte Abgrenzungsprobleme auf, weil z.B. die mit dem Aushandlungskonzept zunächst assoziierte Symmetrievoraussetzung der Interaktionsrollen häufig gar nicht gilt; ebenso ist aber auch die konstante Asymmetrie nur ein Sonderfall. Drittens schließlich kann man feststellen, daß sehr unterschiedliche Entwicklungsformen für die Herstellung gemeinsamer Positionen vorkommen, die durch die Dimensionen 'Schrittweite' und 'Festgelegtheit' bestimmt sind; der mit dem Aushandlungskonzept verbundenen Vorstellung eines Austausches von Position und Gegenposition stehen also beispielsweise Verfahrensformen gegenüber, wo die Praktikabilität möglicher Positionen gemeinsam 'durchgespielt' werden. Trotz dieser Schwierigkeiten möchte ich im vorliegenden Beitrag bei der Verwendung des Aushandlungsbegriffs - für den ich vorläufig keinen Ersatz sehe - bleiben und ihn dementsprechend in einem sehr weiten Sinne verstehen.

Im folgenden will ich nun einige Angaben über das mutmaßliche Grundmuster von Aushandlungen machen; sic basieren auf gemcinsamen Vorarbeiten von W. Kallmeyer und mir und sind teils empirisch, teils theoretisch gewonnen. Zunächst kann man vier (nicht immer explizit realisierte) Phasen bei Aushandlungen unterscheiden: Auslösung, Vorbereitung, Durchführung und Abschluß. In diesen vier Phasen werden jeweils typische Aktivitäten durchgeführt, die ich hier - ohne Anspruch auf Vollständigkeit grob skizzieren möchte.

Auslösung: Anlaß für die Durchführung eines Aushandlungsverfahrens ist, daß mindestens einer der Interaktionsbeteiligten die Existenz eines Positionsproblems annimmt (oder aus strategischen Gründen anzunehmen vorgibt), das die Lösung der zugrundeliegenden Interaktionsaufgabe behindert. Ein derartiges Problem kann z.B. darin bestehen, daß zu einem relevanten Punkt eine Position der Interaktionsteilnehmer fehlt, daß vorhandene Positionen unterschiedlich sind oder daß Zweifel an ihrer (hinreichenden) Übereinstimmung bestehen etc. Die entsprechende Existenzannahme ist explizit zu markieren und gegebenenfalls der Problemtyp anzudeuten.

Vorbereitung: Es wird geprüft, ob das angenommene Problem besteht (dies wird nicht immer eindeutig entscheidbar sein) oder weiterhin als existent 
angenommen werden soll. Wenn dies der Fall ist, wird eine Entscheidung darüber getroffen, ob eine Behandlung des Problems zum gegenwärtigen Zeitpunkt erforderlich oder überhaupt erfolgversprechend ist. Im negativen Fall wird eine Behandlung aufgeschoben (oder nach Änderung der globalen Interaktionsaufgabe) für unnötig erklärt. Im positiven Fall ist demgegenüber ein Aushandlungsziel zu definieren und ein Verfahrenstyp zu wählen.

Durchführung: Hier sind unterschiedliche Aktivitätsebenen voneinander zu unterscheiden. Erstens werden Positionsdefinitionen durch Angebot (oder Gegenangebot), Übernahme, Zurückweisung, Aufrechterhaltung (Insistieren), Korrektur von Positionen vorgenommen. Zweitens werden Positionen bewertet durch Präferenzmarkierungen oder durch Begründungen ihrer Zweckmäßigkeit bzw. Notwendigkeit. Drittens wird über Positionen entschieden durch Überprüfung der Voraussetzungen und Konsequenzen ihrer Übernahme sowie durch Gewichtung der verschiedenen Beurteilungsaspekte.

Abschluß: Die Übernahme bzw. Nichtübernahme einer gemeinsamen Position wird als Ergebnis formuliert und die Tatsache, zu diesem Ergebnis gekommen zu sein, wird bewertet. Schließlich muß die Rückleitung in den übergeordneten interaktiven Zusammenhang vorgenommen werden.

Eine besonders wichtige Aufgabenstellung bei der genaueren empirischen Bestimmung des Grundmusters von Aushandlungen und seinen Aktivitätstypen besteht darin, daß nach stereotypen sprachlichen Realisierungen dieser Aktivitätstypen gesucht werden muß. Dieser Punkt ist deshalb so wichtig, weil die Erkennung und Identifizierung von Aktivitätstypen erleichtert bzw. objektiviert wird, wenn man für eine Identifizierung - statt ausschließlich interpretative Kategorisierungen aufgrund der eigenen Sprachintuition einzusetzen - auf 'formale' Indikatoren zurückgreifen kann. Tatsächlich ist bei Überprüfung größerer Materialmengen relativ schnell ersichtlich, daß es z.B. typische grammatische Figuren gibt, mit denen sich die Gesprächsteilnehmer selbst gegenseitig den jeweils intendierten Aktivitätstyp identifizierbar machen. Relativerend zu dem bisher Gesagten muß angemerkt werden, daß Aushandlungen nicht stets genau in der Form des oben skizzierten Musters vorkommen, sondern daß es je nach Kontext sehr unterschiedliche Ausprägungen davon gibt. So bedarf es z.B. für den Fall der Bedeutungsaushandlungen in der Regel keiner expliziten Vorbereitungsphase: das Ziel der Herstellung von Bedeutungsgleichheit kann in den meisten Kommunikationskontexten als gemeinsam unterstellt werden und auch im Fall der therapeutischen Kommunikation ist das Ziel der Deutungsangleichung bereits institutionell vorgegeben (letzteres kann allerdings als nur langfristig erreichbar gelten). Neben der Möglichkeit, daß das Aushandlungsmuster unterschiedlich vollständig realisiert ist, besteht ein weiterer, zentraler Grund für die Existenz von Ausprägungsvarianten in kontextabhängigen Unterschieden der Beteiligungsrollen von Interaktanten; speziell ist hier die Fähigkeit oder Zulässigkeit der Finhringung von Instanzenwissen zur Durchsetzung einer Position von großer Bedeutung. Dabei sind 
beispielsweise im Falle der Aushandlung von Bedeutungen die Verhältnisse wesentlich komplizierter, als durch die übliche Einteilung von Kommunikationssituationen in symmetrische und asymmetrische erfaßt werden könnte. Welche Instanz für die Interpretation von Äußerungen qua Sprachoder qua Weltwissen verantwortlich ist, das kann von Äußerung zu Äußerung wechseln und deshalb gibt es auch für die Rolle des Autoritätsträgers sehr unterschiedliche Zuordnungen. Hinzu kommt noch, daß unterschiedliche Instanzen miteinander konkurrieren können; im Fall der Äußerungsbedeutung sind dies insbesondere sprachliche Konventionen gegenüber der Sprecherintention [1]. Insgesamt gesehen muß man also möglicherweise mit sehr unterschiedlichen Dominanzverhältnissen und deren Auswirkungen auf die Musterrealisierung von Aushandlungen rechnen.

\section{Deutungsaushandlungen}

In diesem Abschnitt möchte ich aufzeigen, wie das Aushandlungskonzept zur Analyse des interaktiven Umgangs mit Deutungen eingesetzt werden kann, und zugleich sollen einige Spezifika von Therapiegesprächen, die sich bei einer derartigen Analyse ergeben, vorgestellt werden. Zuvor müssen allerdings einige Bemerkungen über Rahmenbedingungen von Deutungen in Therapiegesprächen gemacht werden.

Die Qualität der Analyse eines konkreten Therapiegesprächs hängt wesentlich davon ab, wieviel Wissen über das im zugrundeliegenden kommunikativen Muster bzw. über Muster von Gesprächsteilen bereits zur Verfügung steht. Unbeschadet davon, daß Patienten wie Therapeuten im Prinzip sehr unterschiedliche Kommunikationsmuster in Therapiegespräche einbringen können, wird man nämlich davon auszugehen haben, daß die Gesprächsteilnehmer für die Durchführung eines Therapiegesprächs jeweils auf ein begrenztes Repertoire von alltagsweltlichen Standardmustern zurückgreifen (im Falle einer länger andauernden Therapie werden solche Muster allerdings spezifischen Umformungen ausgesetzt sein). Insofern ist es wichtig, die Muster

[1] Nebenbei bemerkt wird es mit Hilfe des Aushandlungskonzepts möglich, einen alten Streit in der sprachphilosophischen Diskussion zwischen den sogenannten Intentionalisten und den Konventionalisten zu entscheiden bzw. ihn als empirisch unangemessen einzuschätzen. In der Kommunikation besitzt weder die Sprecherintention noch die konventionelle Bedeutung eine absolute Präferenz, sondern zwischen ihnen besteht ein Aushandlungsspielraum. Die Ähnlichkeit oder Übereinstimmung der Bedeutungszuordnung kann idealisierend aufgrund von Sprachkonventionen unterstellt werden, aber es besteht in der Regel auch die Bereitschaft, bei Bedeutungsdifferenzen die Sprecherintention oder die Hörerbedeutung temporär zu übernehmen, sofern der Abweichungsgrad nicht $\mathrm{zu}$ groß ist. 
von therapeutischer Kommunikation $\mathrm{zu}$ kommunikativen Mustern in vergleichbaren Situationen (z.B. Beratungsgespräche, Problemlösungsgespräche) in Beziehung zu setzen. Auf diesen Punkt kann ich hier allerdings nicht genauer eingehen und es soll hier der Hinweis genügen, daß Therapiegesprächen mit derartigen Gesprächstypen gemeinsam ist die Unterteilung in Phasen mit der dominanten Funktion einer Informationseinholung gegenüber Phasen, in denen eher die gewünschten Konsequenzen gezogen und diskutiert werden. Diese beiden Typen von Phasen korrespondieren mit dem Einsatz spezifischer Gesprächstechniken: für die Phase der Informationseinholung ist dies beispielsweise u.a. die Technik von Frage-Antwort-Sequenzen. Je nach Phase sind natürlich auch die Beteiligungsrollen der Gesprächspartner unterschiedlich definiert und dementsprechend ist speziell der Umfang der Redeanteile im allgemeinen unterschiedlich groß; so wird z.B. der Redeanteil des Patienten in einer Phase der Informationseinholung in der Regel größer sein als der Anteil des Therapeuten, während in einer Deutungsphase die Anteile ungefähr gleich groß sind oder der Anteil des Therapeuten sogar höher liegt. Die Charakterisierung einer Sequenz eines Therapiegesprächs als Phase der Informationseinholung schließt auch nicht aus, daß dort bereits konsequenzbildende Aktivitäten, also insbesondere Deutungen, vorkommen; entsprechende Charakterisierungen basieren nämlich nur auf Dominanzverhältnissen.

Wie oben schon angedeutet ergeben sich im Kontext von Therapiegesprächen Sonderbedingungen für die Realisierung des Aushandlungsmusters bezogen auf Deutungen. In diesem Kontext bedarf es im allgemeinen keiner manifesten Legitimationsbemühungen mehr dafür, daß der Therapeut Deutungen hervorbringt, wenn er sie für explizierungswürdig hält oder wenn er Zweifel hat, ob der Patient ihnen zustimmt. Ebenso ist es selbstverständlich, daß der Patient nicht von vornherein das Erfordernis oder die Möglichkeit einer erfolgreichen Behandlung der eingebrachten Deutungen in Frage stellt, sondern daß er entweder seine Übereinstimmung mit der jeweiligen Deutung signalisiert oder unmittelbar in die Aushandlung über sie eintritt (und damit implizit sein Einverständnis zu einer solchen Aushandlung gibt). Insofern ist zu erwarten, daß von Deutungsaushandlungen in Therapiegesprächen hauptsächlich die Durchführungsphase manifest vorliegt; daher möchte ich mich im folgenden auch auf die Diskussion der Aktivitätstypen in dieser Phase beschränken.

Im Sinne des eben Gesagten kann vereinfacht das Einbringen einer Deutung (sei es durch den Therapeuten oder durch den Patienten selbst) als Beginn einer Aushandlung über sie gewertet werden. Damit ergibt sich als Minimalstruktur für eine Deutungsaushandlung ein Äußerungskomplex bestehend aus einer Deutung eines Gesprächspartners und der nachfolgenden Reaktion des anderen Partners. Derartige Minimalstrukturen sind natürlich nur von geringem Untersuchungsinteresse; die Aufmerksamkeit gilt vielmehr längeren 
zusammenhängenden Äußerungskomplexen mit einer Deutung als Anfangspunkt [2].

Wenn man aushandlungseinleitende Deutungen im Material überprüft, dann fallen zwei Spezifika auf. Einerseits sind solche Deutungen - zumindest von seiten des Therapeuten - häufig relativ 'stark' (im Sinne von Abschnitt 2.), d.h. im Vergleich $\mathrm{zu}$ anderen Kontexten wird relativ viel Hintergrundwissen und speziell psychologisches Wissen 'unvermittelt' eingesetzt. Ein Grund für die Wahl starker Deutungen kann in der angestrebten Effektivität des Therapiegesprächs liegen, damit verbunden ist aber die Gefahr des Aufbaus von Widerständen. Andererseits fällt der durchgängige Angebotscharakter der einleitenden Deutung auf, der durch typische sprachliche Realisierungen angezeigt wird. Daß Deutungen angeboten und nicht schon als festgeschrieben dargestellt werden, verweist bereits auf den in der Regel zugrundeliegenden Aushandlungstyp. Deutungsaushandlungen werden in diesem Fall definiert und/oder praktiziert als die Entwicklung einer gemeinsamen Position der beiden Gesprächspartner und in diesem Sinne kann man sie als 'offen' bezeichnen. Näher betrachtet muß allerdings genau zwischen verschiedenen möglichen Arten von Offenheit unterschicden werden: auf der Ebene der Situationsdefinition kann Offenheit aus therapieideologischen Gründen signalisiert werden, ohne zugleich praktiziert zu werden; sie kann aber auch aus therapiestrategischen Gründen kurzfristig und partiell praktiziert werden, um das zugrundeliegende Ziel des Therapeuten zu verschleiern, seine schon von Beginn an vorhandenen und feststehenden Deutungen langfristig durchzusetzen. Lokal eröffnet aber der Angebotscharakter in jedem Fall die Möglichkeit zu Rückzugspositionen für den Deutenden (dies ist speziell im Falle von 'starken' Deutungen wichtig) und zugleich wird für den Gesprächspartner der Spielraum für die Konkretisierung der eigenen Deutungspositionen geschaffen (vgl. hierzu auch Meyer-Hermann und Weingarten (1982)). Für die stereotype verbale Realisierung des Angebotscharakters von Deutungen möchte ich wegen ihres linguistischen Interesses einige Beispiele angeben.

Bei Deutungsangeboten fällt eine spezifische Stereotypie in folgenden vier Ebenen auf: bei der Anzeige der Konsequenzbildung; bei der Formulierung des Geltungsanspruches; bei der Charakterisierung des Konsequenztyps und bei der Anzeige der Formulierungsschärfe. Die Konsequenzbildung wird häufig durch Phrasen wie also, dann, das heiBt angezeigt. Der Geltungsanspruch der in einer Deutung enthaltenen Sachverhaltsbehauptung kann im Hinblick auf Herkunft und Sicherheit näher spezifiziert sein: Gefühl (ich habe das Gefühl), Glaube (ich glaube, ich meine) oder Möglichkeit (vielleicht, kann es nicht

[2] Die nachfolgend zusammengefaßten Beobachtungen basieren auf einer Auswertung veröffentlichter Ausschnitte von Therapiegesprächen in Flader (1979), Frankenberg (1979), Goeppert und Goeppert (1979), Koerfer und Neumann (1982), Meyer-Hermann und Weingarten (1982), Schröter (1982) und Weingarten (1982). Der gebotenen Kürze dieses Beitrags wegen kann ich die zugehörigen Belegstellen leider nicht einzeln aufführen. 
sein...?). Gelegentlich wird ein für therapeutische Deutungen wichtiger, spezieller Konsequenztyp explizit angezeigt, der auf 'tieferliegende' Ursachen hinweist: typische Wendungen hierfür sind eigentlich, im Grunde genommen. Schließlich wird sehr häufig - speziell in der Gesprächstherapie - durch bestimmte 'Vagheitsmarkierer' die Formulierungsschärfe eingeschränkt: genannt seien hier die Formulierungen ein bißchen, irgendwie, so, oder so, oder irgendwie.

In gleicher Weise wie eben vorgeführt sind auch für andere Aktivitätstypen in Deutungsaushandlungen zugehörige typische sprachliche Realisierungen angebbar. Das Akzeptieren eines Deutungsangebots geschieht im einfachsten und wohl häufigsten Fall durch eine explizite Bejahung, wobei diese auch durch Deutungsergänzungen oder Begründungen expandiert wird; daneben gibt es aber auch viele indirekte Formen des Akzeptierens. Analoges gilt für (strikte) Zurückweisungen von Deutungsangeboten. Für Aussagen über die sprachliche Realisierung interessanter sind die in vielfältigen Schattierungen vorkommenden Zwischenformen zwischen Akzeptieren und striktem Zurückweisen (verbalisiert z.B. durch in gewisser Weise wohl, also nicht hundertprozentig, na ich weiß nicht, ob dat nich $n$ Tick überinterpretiert is, we'mer das so will, ja aber... in anderer Hinsicht). Häufig ist auch die Aktivität des Insistierens auf Deutungen oder auf Zurückweisungen von solchen zu beobachten; hierzu wird insbesondere das Mittel der sprachlichen Verstärkung durch Wiederholung, Paraphrasierung, Präzisierung etc. verwendet. Dabei ist interessant, daß im Falle konträrer Positionen zu Deutungen von den Beteiligten in der Regel offensichtlich kein Zwang zur Einigung gesehen wird; konkret heißt dies, daß lokales Insistieren auf Deutungen nur selten und eigentlich nie über mehrere Stufen hintereinander vorkommt und daß dementsprechend erfolglose Aushandlungen (sogar ohne explizite Markierung) abgebrochen werden.

Als spezielle Beobachtungen bei Deutungsaushandlungen möchte ich noch drei Punkte ansprechen. Zunächst soll im Sinne der einleitenden Bemerkungen noch einmal die Frage aufgegriffen werden, ob spezifische semantische Aktivitäten in Therapiegesprächen beobachtbar sind. In dem mir zugänglichen Material fallen diesbezüglich zumindest gelegentliche Wortbedeutungsaushandlungen auf, die sich auf alltagssprachliche Wörter beziehen und deren Präzisierungsanspruch mir über dem in anderen Kontexten üblichen Niveau zu liegen scheinen. Hierfür möchte ich ein Beispiel angeben, wo eine Klientin $I$ der Beraterin $B$ über selbst auferlegte Geduldsproben berichtet.

$B$ : Das gibt dir ja dann auch ein Erfolgserlebnis, wenn du das geschafft hast, durchzuhalten.

I: Ja, ich sagte vorhin schon, daß ich vielleicht den Fehler mache, daß ich immer 7.11 hohe Anforderungen stelle. Das kommt jetzt auf die Begrifflichkeit drauf an, Erfolgserlebnis, wo fängt das an, wo hört auf, es ist 
zumindestens so das Gefühl, daß ich anschließend ins Bett gehe und nicht mehr unzufrieden bin, sondern sag ha kannst nen Haken hinter Tagen äh Tagespunktordnung machen, den Punkt hast du auch absolviert, ne

B: Mhm

I: Es g kommt nicht mal drauf an, wie ich es gemacht habe, sondern ich hab es geschafft, auch das

$B$ : du hast nicht abgebrochen und so

I: Mhm

B: Mhm

I: Das ist nicht unbedingt hm $\mathbf{n}$ Erfolgserlebnis in gewisser, du bist die Größte, du hast das wunderschön gemacht, aber ähm ich hab es geschafft doch noch, was ich morgens nicht glaubte, hab ich abends dann doch noch, einfach nur auf, sagen wir auch so von der Zeit her und von der Geduld her geschafft, ne

$B$ : Aber richtige Erfolgserlebnisse dir einzugestehen, das fällt dir schwer

Die letzte Äußerung von $B$ zeigt, daß sie die von $I$ vorgeschlagene Bedeutungsabgrenzung für 'Erfolgserlebnis' akzeptiert hat; anschließend wird das problematisierte Wort mehrfach im präzisierten Sinne verwendet.

Ein zweiter in Deutungsaushandlungen auffallender Punkt ist ein Phänomen, das ich 'Deutungsportionierung' nennen möchte. Der Therapeut hat aufgrund einer vom Patienten geschilderten Sachlage eine etwas kompliziertere Deutung anvisiert und zerlegt diese Deutung in kleine Portionen, die er dem Patienten in einer bestimmten Reihenfolge anbietet und deren Ratifizierung er jeweils abwartet, bevor er eine neue Deutungsportion hinzufügt (vgl. hierzu z.B. dic Sequenzen 119-137 in Frankenberg (1979: 64), und 19-21 in Gocppert und Goeppert (1979: 107)). Die dritte und letzte zu erwähnende Beobachtung schließt an die oben geführte Diskussion der Rollenverteilung in Deutungsaushandlungen an. Relativ häufig kann man Deutungsaushandlungen finden, in denen die Deutungsentwicklung kooperativ gehandhabt wird, d.h. insbesondere der Patient führt oft begonnene Deutungen des Therapeuten fort. Seltener scheint demgegenüber der Fall vorzukommen, daß der Therapeut eine Selbstdeutung des Patienten fortsetzt.

$\mathrm{Daß}$ der hier skizzierte theoretische Rahmen und die mitgeteilten Beobachtungen über Deutungsaushandlungen für eine Analyse von Therapiegesprächen relevant sein können, dürfte einleuchtend sein, und ich werde in den nächsten beiden Abschnitten versuchen, diesen Anwendungsaspekt zu konkretisieren. Die in Deutungsaushandlungen erkennbaren Aktivitäten und Verfahren sind aber in ähnlicher Weise auch bei Aushandlungen von Sprachbedeutungen zu beobachten und insofern zeigt sich die eingangs postulierte Relevanz der Untersuchung therapeutischer Kommunikation für die Semantik. Ich $\mathrm{muß}$ es in diesem Beitrag allerdings bei der pauschalen Behauptung belassen, daß erst eine Kenntnis der zur Konstruktion von Bedeutungen 
eingesetzten Verfahren den Semantikern dazu verhelfen wird, die bisher weit unterschätzte 'kreative' Dimension von Interpretationen zu erfassen (vgl. hierzu aber Kindt (1984b)).

\section{Exemplarische Analyse}

In diesem Abschnitt soll anhand eines längeren Gesprächsausschnittes demonstriert werden, wie die im vorangegangenen eingeführten Kategorien zur Beschreibung und Analyse von therapeutischer Kommunikation eingesetzt werden können. Der Gesprächsausschnitt stammt aus einem ersten längeren Beratungsgespräch, das die Beraterin Bärbel $(B)$ mit der Studentin Ingrid $(I)$ führt. Ingrid ist fünfundzwanzig Jahre alt und lebt mit ihrem Freund Felix zusammen. Nach Behandlung unterschiedlicher Themen zu Beginn des Beratungsgesprächs kommt Ingrid plötzlich auf ihre Mutter zu sprechen ('muß ich jetzt schon lachen, sofort in meinem Hinterkopf tickt meine Neurose gegenüber meiner Mutter'). Das folgende Gespräch über die Mutter hat zunächst vorwiegend informierenden Charakter. Dabei schildert Ingrid, daß ihre Mutter ständig an ihr auszusetzen hat oder hatte; trotz dieser überhöhten Anforderungen der Mutter gelingt es Ingrid nicht, sich von ihr abzulösen. Weiterhin stellt Ingrid dar, wie sie und ihr Partner Felix unter Druck gesetzt werden, wobei insbesondere der Wunsch der verwitweten Mutter, umsorgt zu werden und nicht allein sein zu müssen, eine Rolle spielt. Schließlich gibt Ingrid deutlich ihre negative Bewertung der Mutter zu erkennen: 'menschlich gesehen verurteile ich sie furchbar'; 'ich ekle mich teilweise vor ihr'; 'das kotzt mich also furchtbar an'; 'ich finde nichts Positives an meiner Mutter'; 'meine Mutter ist für mich so ein rotes Tuch'.

Mit der ersten Äußerung im nachfolgend wiedergegebenen Ausschnitt beginnt eine Deutungsphase; dabei knüpft Bärbel einerseits an Ingrids negative Bewertung der Mutter an und stellt andererseits eine Beziehung zu dem Thema des 'angekratzten' Selbstbewußtseins her, das vor dem Gespräch über die Mutter im Zusammenhang mit anderen privaten und beruflichen Problemen behandelt worden war.

$B$ : (1) Du bist im Grunde genommen ziemlich wütend auf deine

(2) Mutter, daß sie mit die zumindest mit diejenige war, die

(3) dafür gesorgt hat, daß dein Selbstbewußtsein nicht so

(4) ist, wie du es gerne hättest.

I: (5) Nee nee Bärbel nee, ich weiß daß sies bestimmt mit war, ich bin

(6) nicht wütend auf sie, weil ich manchmal doch jetzt schon in

(7) letzter Zeit durch mein Privatleben in die Situation der Mutter-

$B:\left[\begin{array}{l}(8) \\ (9)\end{array}\right.$

verstehst sie 
I: (10) Sie is so und sie wird auch noch so in zwanzig Jahren sein, wann wie

(11) sollte sie sich denn ändern, wie sollte sie sich denn zeigen

(12) wenn das ihre Persönlichkeit ist

$B$ : (13) Das ist für dich unvorstellbar

$[(14)$ daß sie sich ändert

a, das hab ich aufgegeben, da kann

(16) ich äh da kann ich

$B:$ (17) Und du akzeptierst sie auch in ihrer Persönlich-

(18) keit, du meinst ja (...)

$I:[(19) \quad$ Ich weiß nicht was akzeptieren ist

$B$ : (20) mein ich insofern, du sagst dir, sie is halt so man kann sie

[(21) nich mehr ändern und

I: $\lfloor(22)$

kannst

$B$ : (23) sie nicht ertragen, aber du versuchst irgendwie, sie zu verstehen

[(24) ihre Situation ihre Lage

$I:[(25)$

Ich verstehe sie heute ein bißchen besser

(26) als wie mit siebzehn, aber ich tolerier sie nicht, ich kann

$B:[(27)$ $\mathrm{mhn}$

I: (28) sie nicht akzeptieren, aber ich versuch mich ihr gegenüber zu

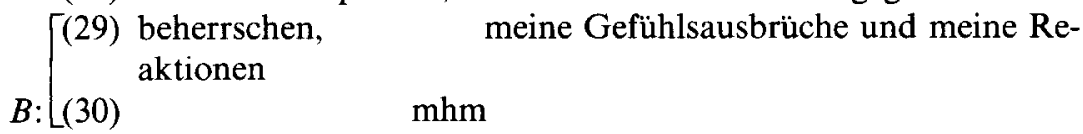

$I:[(31)$ zu beherrschen, naja das is dasselbe

$B:[(32) \quad$ Du versuchst auf jeden Fall, ihr nicht wehzu-

[(33) tun, ne ihre Lage irgenwie nicht noch schlimmer zu machen

I: (34) ja Ja, aber

(35) ich merke, daß ich mir in demselben Augenblick wenn ich ihr nicht

(36) wehtue, mir selber furchtbar wehtue und vielleicht einem anderen

(37) wie Felix oder so, weil wir uns dann so unwürdig menschlich unwür

$B:[(38)$ mhm

I: (39) dig unterbuttern lassen, und daß es uns menschlich ganz schön

(40) an die Substanz geht, ne

$B:[(41)$

Aber du hast vielleicht das Gefühl, daß

(42) du das besser verkraften kannst und Felix als sie (..) ist es

I: (43)

Das weiß ich nicht

$B:[(44)$ deshalb

I: (45) An sich könnt es von von der Struktur her könnt es mcinc

(46) Mutter wesentlich besser verkraften, weil sie sehr dickfellig ist

(47) und sich kaum Gedanken über so was macht

$B:\lfloor(48)$

Hm, aber du kannst

(49) es dir nicht vorstellen, daß du den Kontakt zu ihr total abbrichst $[(50) \ldots$

$I:[(51)$ Nein, weil ich das schon $\mathrm{x}$-mal exerziert hab, dabei geh ich aufm 
(52) Zahnfleisch, das is ja das Merkwürdige

$B:[(53)$

Du machst dir dann

$I:\left[\begin{array}{l}(54) \text { Vorwürfe } \\ (55)\end{array}\right.$

Nee, ich vermisse sie, ich fange sie an nach einiger Zeit

(56) zu vermissen, ich fang irgendwas an, ich war da schon mal beim

(57) Arzt, ich ich hab mit siebzehn Selbstmord gemacht im Internat

(58) und äh, was heißt äh Selbstmord, ist eigentlich klingt ein bißchen

(59) witzig, ne, was äh hab ich ganz simpel gemacht,

ähm ja,

$B:(60)$

Du hast es versucht

I: (61) ich hab Schlafpillchen genommen, kann ich heute nur drüber lachen, ne [3]

Der Gesprächsausschnitt soll zunächst als Realisierung einer Deutungsaushandlung charakterisiert werden. $\mathrm{Zu}$ Beginn bietet Bärbel eine 'Tiefendeutung' (im Sinne von Abschnitt 4) an, die eine vermutete tieferliegende Ursache für die negative Einstellung von Ingrid ihrer Mutter gegenüber benennt. Ingrid weist dieses Deutungsangebot zurück (Zeile 5-6) und gibt eine Plausibilisierung für die Zurückweisung an (Zeile 6-8). Diese Plausibilisierung besteht aus zwei Elementen: einerseits deutet Ingrid eine partielle Fähigkeit an, die Perspektive der Mutter mit ihrer Spezifik der Mutterrolle übernehmen zu können (der in kooperativer Fortführung von Bärbel antizipierte Sachverhalt des Verstehens wird von Ingrid allerdings nicht ratifiziert); andererseits stellt Ingrid die von ihr negativ bewerteten Verhaltenweisen als stabile Persönlichkeitsmerkmale dar, die nicht mehr veränderbar sind. Beide Plausibilisierungselemente unterstellen eine Interpretation von 'wütend', die ausschließt, daß man auf jemanden wegen Verhaltensweisen wütend sein kann, über die er nicht (mehr) frei verfügt. Ein Aspekt des Deutungsangebots von Bärbel wird allerdings durch Ingrid akzeptiert bzw. sogar in der Sicherheit seiner Gültigkeit verstärkt, nämlich die Annahme, daß die Mutter eine Mitverantwortung für das mangelnde Selbstbewußtsein von Ingrid trägt.

Bei der nachfolgenden Äußerung von Bärbel (Zeile 13-14) bleibt vorerst unklar, ob Bärbel die Zurückweisung von Ingrid bereits akzeptiert hat oder ob sie einen Widerspruch gegen die Zurückweisung aus strategischen Gründen nur vorläufig zurückstellt. Demgegenüber wird m.E. deutlich erkennbar, daß Bärbel in der Folge den Typ einer 'offenen' Deutungsaushandlung praktiziert und dabei auch die Strategie der Portionierung anwendet, indem sie jeweils für einzelne Aspekte der Einstellung von Ingrid zu ihrer Mutter kleinschrittig Deutungen anbietet und unter Kooperation von Ingrid die Entwicklung eines Gesamtbildes fördert. Bärbel beginnt mit einem paraphrasierenden Rückgriff

[3] Transkriptionserläuterungen: [wird benutzt als Zeichen für Überlappung bzw. Sprechanschluß; (...) als Zeichen für unverständliche Stellen auf dem Tonband. Pausen und Intonation sind nicht repräsentiert; das Kommazeichen dient nur der Gliederung zur Erleichterung des Lesens. 
auf die vorherigen Äußerung von Ingrid. Diese Art des Beginns ist m.E. insofern typisch, als hier - wie oft - von der Möglichkeit Gebrauch gemacht wird, an den Anfang einer Deutungsentwicklung eine unproblematische und damit leicht akzeptierbare Deutung zu setzen. So bleibt die Ratifizierung durch Ingrid auch nicht aus (Zeile 15). Der Status des zweiten Angebots (Zeile 17-18) als Fortsetzung einer begonnenen Portionierung wird daran erkennbar, daß Bärbel Ingrid unterbricht und die Kontinuitätsindikatoren 'und', 'auch' benutzt; im Vorgriff auf das folgende sei an dieser Stelle bereits angemerkt, daß Kontinuität durch die wiederholte Übernahme einundderselben syntaktischen Figur hergestellt wird ( $D u$ versuchst auf jeden Fall, aber Du hast vielleicht das Gefühl, aber Du kannst es Dir nicht vorstellen, Du machst Dir dann Vorwürfe ). Die Behandlung der zweiten Bedeutungsportion wird unterbrochen durch eine kurze Aushandlung über die Bedeutung des Wortes 'akzeptieren'; obwohl Ingrid kurz vor Beginn des wiedergegebenen Ausschnitts selbst das Wort 'akzeptieren' in Bezug auf ihre Mutter gebraucht hat (weil ich mir vom Verstand her sage, es gibt bestimmt irgendwas, worin Du sie schätzen und akzeptieren könntest), gibt Ingrid hier vor, die Wortbedeutung nicht zu wissen. Aus der Art, wie Ingrids Problematisierung in folgenden behandelt wird, kann man aber schließen, daß Ingrid eher eine Bedeutungsdifferenz zwischen ihr und Bärbel vermutet und bei Zugrundelegung ihrer eigenen Interpretation von 'akzeptieren' das Deutungsangebot von Bärbel zurückweisen würde (dies tut sie später auch vgl. Zeile 27-28). Die Unterstellung einer möglichen Bedeutungsdifferenz führt Bärbel dazu, ihre Bedeutungsintention zu explizieren (Zeile 20-21). Mit der Äußerung in Zeile 22 geht Ingrid bereits wieder in Deutungsaushandlung zurück; implizit hat sie damit (zumindest lokal) die Bcdcutungsexplikation von Bärbel und damit die Sprecherintention als Instanz ratifiziert. Zugleich akzeptiert Ingrid implizit das Deutungsangebot von Bärbel relativ zur ausgehandelten Bedeutung, indem sie sich negativ von einer anderen möglichen Deutung abgrenzt (elliptische $j a$, aber-Figur). Bärbel ratifiziert diese Abgrenzung (Zeile 21, 23) und versucht, die Deutungsentwicklung wieder durch eine positive Charakterisierung der Einstellung von Ingrid zu ihrer Mutter fortzuführen. Dieses Deutungsangebot wird von Ingrid partiell akzeptiert (Zeile 25-26) und zeitlich relativiert; Ingrid verweist hier bereits auf die Situation in ihrer Jugendzeit, in der sie einen Selbstmordversuch gemacht hat. Der Gefahr einer zu weit gehenden Schlußfolgerung aus dem partiellen Akzeptieren des Deutungsangebots begegnet Ingrid jedoch sogleich dadurch, daß sie selbst Präzisierungsanstrengungen unternimmt: zunächst durch negative Abgrenzungen (Zeile 26, 28), dann durch positive Konkretisierungen (Zeile 28, 29, 31). Interessant an dieser Passage ist erstens speziell, daß Ingrid hier selbst wieder das Wort 'akzeptieren' verwendet, aber jetzt offensichtlich in dem stärkeren Sinne von 'tolerieren'. Zweitens werden bestimmte allgemeine Prinzipien der Deutungs- bzw. Bedeutungsaushandlung im Hinblick darauf deutlich, wie Eingrenzungen vorgenommen werden, nämlich durch einen Wechsel 
von exemplarischen positiven Konkretisierungen und negativen Abgrenzungen.

Nach den bisherigen Ausführungen dürfte das Verfahren der gemeinsamen Deutungsentwicklung in der Folge bereits leicht durchschaubar sein, so daß ich den Rest des Gesprächsausschnitts großflächiger besprechen kann. In Zeile 34 nimmt Ingrid mit Hilfe einer $j a$, aber-Figur eine implizite Zurückweisung vor, deren Inhalt erst aus der nachfolgenden, ausgebauten Plausibilisierung entnommen werden kann: Ingrid hat zwar das Deutungsangebot von Bärbel ratifiziert, daß sie versuche, ihrer Mutter nicht wehzutun; Ingrid weist aber implizit zurk, daß diese Handlungsweise auch angemessen ist, bzw. sie deutet die 'überhöhten' Kosten dieser Handlungsweise für sie und ihren Partner Felix an. Eine typische Art der partiellen Zurückweisung stellt auch Zeile 34, 45 mit der Figur das weiß ich nicht, an sich... dar.

Genau gesehen pendelt die Deutungsentwicklung zwischen der Abhandlung unterschiedlicher Deutungsaspekte hin und her und mit Zeile $48 \mathrm{ff}$. greift Bärbel wieder den Aspekt der Verhaltenskonsequenzen auf, den sie zu Beginn des Gesprächs über die Mutter in der Informationsphase schon einmal explizit als widersprüchlich formuliert hatte. Hier wiederholt Bärbel allerdings paraphrasierend nur das, was Ingrid damals bereits geäußert hatte (ich kann meine Mutter aber nicht fallen lassen). Ingrid ratifiziert diese Paraphrase und liefert eine, noch unkonkret bleibende Plausibilisierung. Die anschließend von Bärbel angebotene Konkretisierungsmöglichkeit (die vermutlich aus alltagsweltlichen Wissenshintergründen stammt) weist Ingrid zurück und initiiert dann selber die Schilderung ihres Selbstmordversuchs als Konkretisierung. Mit dieser Schilderung wird das vorgängige Aushandlungsschema verlassen, was auch durch einen Wechsel der Modalitätsebene vom ernsten Gespräch zur Darstellung im fast 'lustigen' Erzählton angedeutet wird. Kurzfristig kehren Bärbel und Ingrid auch nicht mehr in das abgebrochene Aushandlungsschema zurück.

\section{Diskussion}

Abschließend möchte ich kurz die Frage nach dem Stellenwert einer Analyse wie der vorangegangenen für therapeutische Zwecke ansprechen. Generell gilt, daß durch konversationsanalytische Untersuchungen gefundene Kommunikationsmuster wesentliche kontextuelle Informationen für die Einschätzung einer konkreten, diesem Muster folgenden Gesprächspassage darstellen. Dies wird besonders in solchen Fällen deutlich, wo man die Funktion einer einzelnen Äußerung eines Gesprächsteilnehmers zu bestimmen hat: eine Funktionseinschätzung, die nur auf die lokalen Gegebenheiten dieser Äußerung achtet, bleibt sehr häufig unzureichend oder geht auch leicht fehl, weil diese eventuell gerade eine typische, aber nicht vollkommen explizit manifestierte Musteraktivität realisiert und dann nicht als solche erkannt wird. 
Die Gefahr derartiger Fehlinterpretationen wird dem konversationsanalytisch Geschulten beispielsweise im Rahmen von Kommunikationstrainings bewußt, wie sie in der Regel von Psychologen durchgeführt werden; das kommunikative Argumentations- oder Konfliktverhalten von Teilnehmern in Rollenspielen wird dort nämlich häufig nur aufgrund lokaler Eindrücke bewertet, wodurch weder die Möglichkeit, daß das Rollenspiel unnatürlich ist, in Betracht gezogen und als Grund für das etwa negativ bewertete Verhalten erkannt wird noch die Spezifizität des Verhaltens im Hinblick auf ein 'ordentlich' abgewickeltes Muster in den Blick kommt.

Diese generelle Funktion konversationsanalytischer Untersuchungen kann auch im speziellen Fall der Analyse therapeutischer Kommunikation reklamiert werden. Hinzu kommt in diesem Bereich eine weitere (beim gegenwärtigen Erkenntnisstand aber noch kaum anwendbare) Möglichkeit der Analysehilfe: die Beobachtung von widersprüchlichem kommunikativen Verhalten oder von Abweichungen gegenüber vorgängigen Kommunikationsmustern kann Anlaß für eine gezielte Suche nach zugrundeliegenden, vom Patienten nicht verbalisierbaren Krankheitsursachen sein. Flader und Giesecke (1980) konkretisieren diese Möglichkeit am Beispiel eines psychoanalytischen Erstinterviews, wo die Patientin bei der Schilderung ihrer Krankengeschichte zwei verschiedene Gesprächsmuster so einander überlagert, daß bestimmte auffällige Brüche bzw. Diffusionen entstehen [4].

Beide eben skizzierten Hilfsfunktionen konversationsanalytischer Untersuchungen setzen ausreichende Kenntnisse über jeweilige Kommunikationsmuster voraus. Nun wird jedoch in der gegenwärtigen Methodologiediskussion der Konversationsanalyse nicht einmal die Zielsetzung der Musterbestimmung generell akzeptiert. Und cbensowenig sind die bisher vorliegenden Ergebnisse über Muster durchgängig so spezifisch und zuverlässig, daß höhere Erwartungen an die Güte und Sicherheit darauf aufbauender Interpretationen zu richten wären. Insofern muß 3 auch der folgende und zudem hier nur andeutungsweise ausführbare Versuch, die beiden möglichen Hilfsfunktionen auf den obigen Gesprächsausschnitt $\mathrm{zu}$ beziehen, als vorläufig gelten und dient eher der Problematisierung als der Aufstellung von Interpretationsbehauptungen.

Im obigen Gesprächsausscnnitt sind insbesondere zwei Stellen besonders auffällig. Ich meine einerseits die Zurückweisung von Ingrid (Zeile 5, 6), sie sei nicht wütend auf ihre Mutter, und andererseits den Übergang von Ingrids Aussage, sie vermisse ihre Mutter (Zeile 55), zu der Schilderung ihres Selbstmordversuchs. Bei der ersten Stelle scheint sich eine Widersprüchlichkeit der

[4] Leider bleibt die Interpretation der beiden Autorem im konkreten Fallbeispiel noch sehr problematisch. Grund hierfür ist die unzureichende Bestimmung der zugrundeliegenden Gesprächsmuster und ihrer gegenseitigen Abhängigkeit; ich kann meine diesbezügliche Kritik aus Platzgründen hier aber nicht ausführen. 
Aussagen von Ingrid zu offenbaren: wieso behauptet Ingrid, nicht wütend auf ihre Mutter zu sein, wenn sie doch zuvor gesagt hat, daß sie ihre Mutter menschlich furchtbar verurteile und daß sie das Verhalten ihrer Mutter furchtbar ankotze? Auch später (Zeile 22) äußert Ingrid wieder, daß sie ihre Mutter kaum ertragen könne. Zugleich bleibt die Begründung dafür, daß sie nicht wütend sei, unplausibel: selbst wenn Ingrid das Verhalten ihrer Mutter auf unveränderliche Persönlichkeitsmerkmale zurückführt, kann sie doch auf dieses Verhalten wütend sein. Was muß man aus dieser Widersprüchlichkeit schließen? Wahrscheinlich will Ingrid sich selbst nicht eingestehen oder Bärbel gegenüber nicht zugeben, daß sie in Wirklichkeit doch wütend auf ihre Mutter ist, möglicherweise liegt auch ein Widerstandsphänomen vor; Ingrid wehrt sich gegen eine Aufarbeitung ihrer Gefühle der Mutter gegenüber. Vor einer voreiligen Festlegung auf solche Schlußfolgerungen ist zu warnen, da sie auf Äußerungsinterpretationen aus isolierter Analysandenperspektive basieren, die weder den von den Teilnehmern aufgebauten Kontext noch deren für 'wütend' eingeführte Bedeutungsspezifizierung berücksichtigen. Auf den Aspekt des möglichen Kontexteinflusses kann ich hier nicht eingehen. An dem Gesprächsausscnnitt selbst ist aber belegbar, daß die Aushandlung über die Deutung in Zeile 1-4 indirekt eine bestimmte, interaktiv durchgesetzte Bedeutungsspezifizierung für 'wütend' zur Folge hat. Interaktiv durchgesetzt heißt hier: anläßlich der Begründung Ingrids für ihre Zurückweisung des Deutungsangebots (Zeile $8 \mathrm{ff}$.) gibt es für Bärbel die Reaktionsmöglichkeit, Ingrid Inkorrektheit der Argumentation vorzuwerfen oder die Bedeutung von 'wütend' zu problematisieren; da Bärbel beides nicht tut, gilt bis auf weiteres für 'wütend' eine Interpretation, die die Möglichkeit, wütend zu sein, auf den Fall vorwerfbaren Verhaltens einschränkt. Und relativ zu dieser Interpretation kann eine Widersprühlichkeit der Aussagen von Ingrid nicht behauptet werden. Auch der Umstand, daß evtl. sogar vom Standardgebrauch abweichende Bedeutungsspezifikationen eingeführt werden, ist für die Kommunikation nichts Ungewöhnliches. Insofern können die genannten Schlußfolgerungen durch die Analyse nicht bestätigt werden und sollten daher nicht vorschnell als gültig behauptet werden.

Ein erster Eindruck von der zweiten Stelle (Zeile $48 \mathrm{ff}$.) mag etwa besagen: irgendwie setzt Ingrid ihre Zurückweisung von Bärbels Deutung in Zeile 53, 54 nicht konsequent fort; die Schilderung eines Selbstmordversuchs zu dem - wie man später erfährt - Ingrid sich durch die Verhaltensweise ihrer Mutter getrieben fühlt, kann eigentlich keine Konkretisierung des Umstands sein, daß Ingrid ihre Mutter vermißt. Eine vorschnelle Interpretation würde es hier bei der Postulierung eines Widerspruchs oder Musterabweichung belassen und möglicherweise sogar weitreichende Behauptungen über die Dialektik von Abstoßung und Anziehung in der Mutter-Tochter-Beziehung anschließen. Wieder sind Vorsicht und eine genauere Analyse unter Berücksichtigung der Mustergegebenheiten angebracht. Die Schwierigkeit einer Einschätzung davon, 
wie die Selbstmordschilderung an die vorgängige Deutungsaushandlung anschließt, hängt mit der Vagheit der diesen Übergang repräsentierenden Äußerung von Ingrid zusammen (ich fang irgendwas an, ich war da schon mal beim Arzt ). Zunächst kann man als allgemeine Erfahrung aus konversationsanalytischen Untersuchungen festhalten, daß ein abrupter, vollständiger Ausstieg aus einem vorgängigen Muster nur unter Sondervoraussetzungen möglich ist und daß er dann von den Gesprächsbeteiligten in der Regel durch manifeste Aktivitäten abgesichert werden muß. Da in unserem Fall von Ingrid kein Musterabbruch signalisiert wird, ist zu erwarten, daß der Übergang zur Schilderung des Selbstmordversuchs musterkonform ist. Zu klären bleibt die Logik dieses Übergangs. Eine Interpretationsmöglichkeit, deren Begründung ich hier jedoch nich mehr ausführen kann, würde besagen, daß Ingrid zunächst eine Konkretisierung für ihre Verwunderung über ihr eigenes Verhalten (insbesondere das Vermissen der Mutter) zu geben beabsichtigt und diesbezüglich auf einen Arztbesuch verweist, wo das betreffende Thema 7ur Sprache kam; angesichts der Erinnerung an den Arztbesuch fokussiert sie dann jedoch den primären Anlaß für diesen Besuch, nämlich ihren Selbstmordversuch, und vergißt später die Fortführung der anvisierten Konkretisierung (was durchaus nicht ungewöhnlich wäre).

Unabhängig davon, wie die beiden diskutierten Stellen genau einzuschätzen sind, bleibt abschließend festzuhalten, daß für eine Analyse der Gesprächslogik die Struktur der zugrundeliegenden Deutungsaushandlung berücksichtigt werden muß, wenn man nicht Gefahr laufen will, bestimmte durch gängige Therapieideologien zwar nahegelegte, aber gleichwohl falsche Schlußfolgerungen aus einem scheinbar auffälligen Gesprächsverhalten zu ziehen. Damit soll nicht bchauptet wcrden, daß entsprechende Folgerungen von Therapeuten und ihre darauf aufbauenden Gesprächsstrategien stets und grundsätzlich verfehlt seien; ein entscheidendes Kriterium ist hierbei sicherlich auch, daß nicht vereinzeltes, sondern rekurrentes Gesprächsverhalten die Grundlage für diese Folgerungen bildet. Insgesamt gesehen darf aber von den Ergebnissen konversationsanalytischer Untersuchungen zumindest langfristig erhofft werden, daß sie zu differenzierteren Urteilen darüber führen, ob man von einem bestimmten kommunikativen Verhalten eines Patienten auf psychische Probleme rückschließen kann.

\section{Literaturhinweise}

Flader, Dieter, 1979. 'Techniken der Verstehenssteuerung im psychoanalytischen Diskurs'. In: Flader und Wodak-Leodolter 1979: $24-43$.

Flader, Dieter, Ruth Wodak-Leodolter, Hrsg., 1979. Therapeutische Kommunikation. Königstein: Scriptor.

Flader, Dieter und Michael Giesecke, 1980. 'Erzählen im psychoanalytischen Erstinterview - eine Fallstudie'. In: Konrad Ehlich, Hrsg., Erzählen im Alltag. Frankfurt: Suhrkamp. S. 209-262. 
Flader, Dieter u.a., Hrsg., 1982. Psychoanalyse als Gespräch. Frankfurt: Suhrkamp.

Frankenberg, Hartwig, 1979. 'Gestörte Kommunikation als Reproduktion einer Beziehungsstörung in der Ehetherapie'. In: Flader und Wodak-Leodolter 1979: 44-77.

Freud, Sigmund, 1961. Die Traumdeutung. Frankfurt: Fischer. [1900].

Goeppert, Sebastian, Herma Goeppert, 1979. 'Sprache, Objektbeziehung und Neurose'. In: Flader und Wodak-Leodolter 1979: 98-116.

Kindt, Walther, 1983. 'Neue modelltheoretische Ansätze für die Semantik'. In: Rainer Bäuerle u.a., Hrsg., Meaning, use and interpretation of language. Berlin: de Gruyter. S. 270-289.

Kindt, Walther, 1984a. 'Word semantics and conversational analysis'. In: H.J. Eikmeyer und Hannes Rieser, Hrsg., Words, worlds and contexts. Berlin: de Gruyter. S. 500-509.

Kindt, Walther, 1984b. 'Dynamische Semantik'. In: Burkhard Rieger, Hrsg., Dynamik in der Bedeutungskonstitution. Hamburg: Buske. S. 95-142.

Kindt, Walther, Rüdiger Weingarten, 1984. Verständigungsprobleme. Deutsche Sprache $12: 193-218$.

Koerfer, Armin, Ch. Neumann, 1982. 'Alltagsdiskurs und psychoanalytischer Diskurs'. In: Flader u.a., Hrsg., 1982: 96-137.

Meyer-Hermann, Reinhard, Rüdiger Weingarten, 1982.'Zur Interaktion und interaktiven Funktion von Abschwächungen in Therapiegesprächen'. In: Klaus Detering u.a., Hrsg., Sprache crkennen und verstehen. Tübingen: Niemeyer. S. 242-252.

Schröter, K., 1982. 'Zur Rekonstruktion der Bedeutungsstruktur von Gesprächssequenzen'. In: Dieter Flader u.a., 1982: 194-207.

Weingarten, Rüdiger, 1980. Die Sprache als Gegenstand und Mittel in der klientenzentrierten Gesprächsführung. Diplomarbeit, Universität Bielefeld.

Walther Kindt (b. 1945) took degrees in mathematics and logics. He is teaching linguistics at Bielefeld University and carrying out research projects in formal grammar, empirical semantics and conversational analysis. 